\title{
Earliest detection of the optical afterglow of GRB 030329 and its variability
}

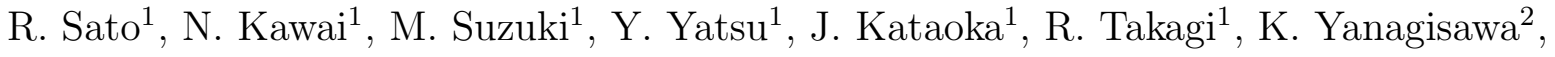 \\ and H. Yamaoka ${ }^{3}$
}

\begin{abstract}
We report the earliest detection of an extremely bright optical afterglow of the gamma-ray burst (GRB) 030329 using a 30cm-telescope at Tokyo Institute of Technology (Tokyo, JAPAN). Our observation started 67 minutes after the burst, and continued for succeeding two nights until the afterglow faded below the sensitivity limit of the telescope (approximately $18 \mathrm{mag}$ ). Combining our data with those reported in GCN Circulars, we find that the early afterglow light curve of the first half day is described by a broken power-law $\left(\propto t^{-\alpha}\right)$ function with indices $\alpha_{1}=0.88 \pm 0.01\left(0.047<t<t_{\mathrm{b} 1}\right.$ days $), \alpha_{2}=1.18 \pm 0.01\left(t_{\mathrm{b} 1}<t<\right.$ $t_{\mathrm{b} 2}$ days $)$, and $\alpha_{3}=1.81 \pm 0.04\left(t_{\mathrm{b} 2}<t<1.2\right.$ days $)$, where $t_{\mathrm{b} 1} \sim 0.26$ days and $t_{\mathrm{b} 2}$ $\sim 0.54$ days, respectively. The change of the power-law index at the first break at $t \sim 0.26$ days is consistent with that expected from a "cooling-break" when the cooling frequency crossed the optical band. If the interpretation is correct, the decay index before the cooling-break implies a uniform ISM environment.
\end{abstract}

Subject headings: gamma-rays: bursts - radiation mechanisms: non-thermal

\section{Introduction}

The overall behavior of GRB afterglows has been successfully explained by the standard fireball model (e.g. Piran et al. 1999). It is expected that the deviation from a simple powerlaw in the afterglow light curve will provide wealth of information on the environment and physical parameters of GRBs. For example, observations of early GRB afterglows confirmed that "breaks" exist in the light curves of a number of GRBs. Such breaks may be understood in the framework of the standard fireball model either as a "jet-break" where the bulk

\footnotetext{
${ }^{1}$ Tokyo Institute of Technology, Meguro-ku, Tokyo, Japan

${ }^{2}$ Okayama Astrophysical Observatory, Asaguchi-gun, Okayama, Japan

${ }^{3}$ Kyusyu University, Ropponmatsu, Fukuoka, Japan
} 
Lorentz factor of the relativistic jet decrease to the inverse of jet opening angle (Sari, Piran \& Halpern 1999, Rhoads 1999), or the "cooling-break" where the high energy electrons start to lose most of their energy rapidly by synchrotron emission which emit the observed optical photons (Sari, Piran \& Nakar 1998). While there are number of convincing cases for jetbreaks, identification of a cooling-break in the optical light curve has been difficult since it requires detection of a subtle change in the power-law index $(\Delta \alpha \sim 0.25)$. In order to study afterglow light curves in detail, continuous coverage and a high signal-to-noise ratio are required.

The situation has been dramatically improved since the advent of High Energy Transient Explorer-2 (HETE-2). HETE-2 can determine the positions of GRBs onboard, and notify ground-based observers of the GRB coordinates within 1-10 minutes after the burst (Ricker et al. 2002). For example, the locations of GRB 021004 (e.g., Fox 2002) and GRB 021211 (e.g., Fox \& Price 2002) were disseminated within less than a minute after the bursts, which prompted detailed studies of GRB afterglows in the very initial phases while they are bright.

GRB 030329 was detected by the HETE-2 satellite on 29 March 2003 at 11:37:14.7 UT. Position was determined by the ground analysis, and the location was reported to GCN at 73 minutes after the burst (Vanderspek et al. 2003). A very $\operatorname{bright}(R \sim 13 \mathrm{mag}$ ) optical transient (OT) was reported at $\alpha=10^{h} 44^{m} 50^{s} .0, \delta=+21^{\circ} 31^{\prime} 17.8^{\prime \prime}(J 2000.0)$ (Peterson \& Price 2003, Torii 2003) inside the SXC error circle. This is the brightest GRB ever detected by HETE-2 with the $30-400 \mathrm{keV}$ fluence of $1.2 \times 10^{-4} \mathrm{erg} \mathrm{cm}^{-2}$, and precise and continuous follow-up observations were carried out by dozens of telescopes distributed around the world. Optical spectroscopic observations have determined its redshift as $z=0.1685$ (Greiner et al. 2003), which is one of the closest ever known and is possibly related to the exceptional brightness of this afterglow. Moreover, spectra taken after several days reveal the evolution of broad peaks in the spectra characteristic of a supernova. The spectral similarity to SN 1998bw (e.g., Galama et al. 1998, Iwamoto et al. 1998) and other energetic supernovae such as 1997ef provides strong evidence that GRB 030329 is associated with core-collapse supernovae (Dado, Dar \& Rujula 2003, Hjorth et al. 2003, Stanek et al. 2003, Kawabata et al. 2003). In order to investigate the kinetic energy of GRB and the immediate vicinity of its progenitor, the early light curve is important. In this letter, we report the earliest detection of the optical afterglow of GRB 030329 starting 67 minutes after the burst.

\section{Observation and photometry}

Our observation was performed at Tokyo Institute of Technology using a 30cm-telescope (Meade LX-200) and an unfiltered CCD camera (Apogee AP6E) equipped with a front- 
illuminated $1024 \times 1024$ CCD chip (Kodak KAF-1001E). The dark current images were subtracted from the obtained CCD images and then flat fielding was applied for all images. We used IRAF/noao/digiphot/apphot/phot packages to analyze the data.

We started observing the preliminary SXC position at 12:44:13 UT on 29 March 2003, 67 minutes (0.047 days) after the burst ${ }^{4}$. The magnitude of GRB afterglow at the very beginning was $R_{c} \sim 12.4 \mathrm{mag}$. This is the earliest detection of the afterglow of GRB 030329 ever reported in literature.

We continued observations for the rest of the night covering $t \sim 0.05-0.30$ days, and performed observations on the following two nights covering the period of $t \sim 0.93-1.21$ days and $t \sim 2.03-2.08$ days, respectively, where $t$ refers to the time since the burst onset. The exposure time of each CCD frame was $10 \mathrm{sec}(0.047<t<1.21$ days; when the after glow was relatively bright) or $30 \mathrm{sec}(2.03$ days $<t)$. The magnitude of the comparison stars were calibrated using three stars in the same field of view, which has been calibrated in detail by Henden. Then we determined the magnitude of the OT in each frame relative to the weighted average of 15 bright comparison stars.

Since the peak sensitivity of our camera is very close to the $R_{c}$ band, we have calibrated magnitude of the OT by converting our magnitude system $\left(R_{\text {inst }}\right)$ into the $R_{c}$ system assuming the color correction with the color $(V-I)=0.74$ mag (Zeh et al. 2003) $\left(a(r)=\left(R_{\text {inst }}\right)-0.1514 \times(V-I)\right.$, where $\mathrm{a}(\mathrm{r})$ is the zero-point between the instrumental system and the standard magnitude). The statistical error is $\sim 0.017-0.02$ mag. Although the zero-point errors were found to be $\sim 0.03 \mathrm{mag}$ for the reference stars by Henden (2003), we found our data were consistently brighter than the $R$-band filtered observations (e.g., Burenin et al. 2003). We therefore introduced additional zero-point correction (+0.11 mag) to match the light curves in the overlapped interval. The resulting light curve of the GRB 030329 afterglow in the $R_{c}$ band is shown by combining data from other observations (see Fig 1).

The light curve in the first day cannot be fitted with a single power-law function. We therefore tried to fit the light curve by two different forms of broken power-law functions. One is given by Beuermann et al.: $F(t) \propto\left[\left(t / t_{b}\right)^{\alpha_{1}^{\prime} n^{\prime}}+\left(t / t_{b}\right)^{\alpha_{2}^{\prime} n^{\prime}}\right]^{-1 / n^{\prime}}$, where $t_{b}$ is the break time, and $n^{\prime}$ provides a measure of the relative width and the smoothness of the break. The other is a "double-broken power-law" function with two breaks with the following form:

\footnotetext{
${ }^{4}$ As a HETE-2 Ops graduate student R. Sato took the initiative and "ran up to the roof to start observing" while the location data were still preliminary.
} 


$$
F(t) \propto \begin{cases}t^{-\alpha_{1}} & \left(t<t_{\mathrm{b} 1}\right) \\ {\left[\left(t / t_{b 2}\right)^{\alpha_{2} n}+\left(t / t_{b 2}\right)^{\alpha_{3} n}\right]^{-1 / n}} & \left(t>t_{\mathrm{b} 1}\right)\end{cases}
$$

where $t_{\mathrm{b} 1}$ and $t_{\mathrm{b} 2}$ are the break times and $n$ provides a measure of the relative width and the smoothness of the break. Here we excluded the "bump" at $t \sim 0.08-0.09$ days, which is discussed in section 3.3.

We found that the former is not acceptable with a reduced $\chi^{2}$ of 1.72 (285 d.o.f) whereas the latter improves the fit significantly (the reduced $\chi^{2} 1.06$ with 283 d.o.f) (see Fig 2).

As a result, it is well described by a broken power-law of the form; $\alpha_{1}=0.88 \pm 0.01$ $\left(0.047<t<t_{\mathrm{b} 1}\right.$ days $), \alpha_{2}=1.18 \pm 0.01$ ( $t_{\mathrm{b} 1}<t<t_{\mathrm{b} 2}$ days $), \alpha_{3}=1.81 \pm 0.04\left(t_{\mathrm{b} 2}<t<1.2\right.$ days), where $t_{\mathrm{b} 1} \sim 0.26$ days and $t_{\mathrm{b} 2} \sim 0.54$ days, respectively, and $n=18.8 \pm 5.1$. Here, $\alpha_{1}$ is determined by essentially the full Tokyo Tech data. The earliest phase of the light curve is well fitted by the single power-law with its index $\alpha_{1} \cdot \alpha_{2}$ and $\alpha_{3}$ are determined by measurements reported by Burenin et al. and GCN (see caption in Fig 1).

\section{Discussion}

\subsection{Light curve at $0.05<t<0.26$ days}

We have presented a light curve of the early phase of the optical afterglow of GRB 030329 starting 67 minutes after the burst. This is the earliest detection of GRB 030329 ever reported (Peterson \& Price 2003, Torii et al. 2003, Uemura et al. 2003).

Burenin et al. (2003) reported follow-up observations of GRB 030329 as early as 6 hours after the burst, using BVRI filters. In each of the filters, they observed a gradual flux decay which can be accurately described as a power-law $F_{\nu} \propto t^{-1.19}$. They also found a characteristic break in the light curve $t_{\text {brk }} \sim 0.57$ day, after which the afterglow flux started to decline faster. The power-law slopes of the light curves changed from -1.19 to -1.9 for $t \geq t_{\text {brk }}$. Notably, this break is nicely consistent with our second break $\left(t_{\mathrm{b} 2}\right)$ within error bars, where the power-law slopes changes from $-1.18 \pm 0.01$ to $-1.81 \pm 0.04$ after the break of $t_{\mathrm{b} 2} \sim 0.54$ days (see above). The first break $\left(t_{\mathrm{b} 1}\right)$ is not discussed in Burenin et al, since they started their observations just around this break $\left(t_{\mathrm{b} 1} \sim 0.26\right.$ days $)$.

Price et al. (2003), Burenin et al. (2003), and Tiengo et al. (2003) found that the results

of their observations are consistent with the model where the afterglow emission is generated during the deceleration of the ultra-relativistic collimated jet. They found that the break in the power-law light curve, at $t \sim 0.5-0.6$ days, can be interpreted as the jet-break, i.e., the 
break which occurs when the angular structure of the ultra-relativistic collimated jet becomes observable (Sari, Piran \& Halpern 1999, Rhoads 1999). This interpretation is supported by the fact that the break occurred simultaneously in different colors. Furthermore, a change in power-law slope from -1.19 to -1.9 , is approximately consistent with that generally observed in jet-break. Therefore, our major concern in this paper is to understand the nature of the first break, $t_{\mathrm{b} 1}$, and examine the consistency between the above scenario in the frame work of standard GRB fireball theories (Piran et al. 1999).

\subsection{Break at $t \sim 0.26$ days}

There are two possible break frequencies in the spectra, $\nu_{\mathrm{m}}$ and $\nu_{\mathrm{c}}$, where $\nu_{\mathrm{m}}$ is the synchrotron frequency, $\nu_{\mathrm{c}}$ is the cooling frequency above which electrons lose their energy rapidly by synchrotron radiation (Sari, Piran \& Narayan 1998). Since $\nu_{\mathrm{m}}$ and $\nu_{\mathrm{c}}$ are the functions of time, a break in the light curve could be observed when $\nu_{\mathrm{c}}$ and/or $\nu_{\mathrm{m}}$ crossed over the observed frequency $\nu_{\mathrm{R}}$. Therefore, we examined six possible cases to understand the first break $\left(t_{\mathrm{b} 1}\right)$, according to the relation between $\nu_{\mathrm{m}}, \nu_{\mathrm{c}}$ and $\nu_{\mathrm{R}}$. In the standard GRB scenario, $\nu_{\mathrm{c}} \leq \nu_{\mathrm{m}}$ is often called "fast cooling" since all electrons cool rapidly, whereas $\nu_{\mathrm{c}} \geq \nu_{\mathrm{m}}$ is referred to "slow cooling" since only the high energy population of electrons cool efficiently. We will also extend our discussion to discriminate between "a homogeneous interstellar medium (ISM) model" (e.g., Sari \& Piran 1999) and "a pre-existing stellar wind model" (e.g., Chevalier \& Li 1999) for the GRB environment. The relationships between observed spectral index and model predictions are compared in Table 1.

We first consider the case where both $\nu_{\mathrm{c}}$ and $\nu_{\mathrm{m}}$ are above the observed optical frequencies $\left(\nu_{\mathrm{R}}<\nu_{\mathrm{m}}<\nu_{\mathrm{c}}\right.$ or $\nu_{\mathrm{R}}<\nu_{\mathrm{c}}<\nu_{\mathrm{m}}$ : case (3) and (6) in Table 1). In these two situations, observed flux at $\nu_{\mathrm{R}}$ should increase with time, which strongly conflicts with the observed declining light curve. On the contrary, if the both cooling frequency $\nu_{\mathrm{c}}$ and the minimum frequency $\nu_{\mathrm{m}}$ are below the optical band $\left(\nu_{\mathrm{c}}<\nu_{\mathrm{m}}<\nu_{\mathrm{R}}\right.$ or $\nu_{\mathrm{m}}<\nu_{\mathrm{c}}<\nu_{\mathrm{R}}$ : case (1) and (4) in Table 1), the predicted optical spectral index would be $\beta=p / 2$, where $p$ is the electron spectral index. Since the photon spectral index of this afterglow was $\beta=0.66$ at $t=0.26$ days (Burenin et al. 2003), we expect $p=1.32$, which is unusually flat for an electron population accelerated in a GRB. Furthermore, power-law index in the light curve should be $\alpha=\frac{2-3 p}{4} \sim 0.49$, which is again inconsistent with $\alpha_{1} \sim 0.88$ obtained with our data.

Case (5) $\nu_{\mathrm{c}}<\nu_{\mathrm{R}}<\nu_{\mathrm{m}}$ in Table 1 is also ruled out since the predicted power-law index $\alpha=0.25$ (Sari, Piran \& Narayan, 1998) is too flat compared to the observed value of $\alpha_{1} \sim 0.88$. Therefore, we argue that the possible solution is $\nu_{\mathrm{m}}<\nu_{\mathrm{R}}<\nu_{\mathrm{c}}$ for the time region of $t \leq t_{\mathrm{b} 1}$. In this case, however, if the burst occurred in pre-existing stellar wind, the 
optical decay slope is predicted to be $\alpha=\frac{3 \beta}{2}-\frac{\delta}{8-2 \delta} \sim 1.49$, with $\delta=2$ for a wind model (Panaitescu, Meszaros \& Rees 1998), which is quite steeper than that observed, and hence we can rule out wind-interaction model. In summary, $\nu_{\mathrm{m}}<\nu_{\mathrm{R}}<\nu_{\mathrm{c}}$ and ISM model (case (2) in Table 1) is the only possible solution to reproduce both the temporal/spectral index of the optical afterglow of GRB 030329 at $0.05<t<0.26$ days.

In such a slow cooling case, time variation of afterglow flux is given by $F \propto t^{-3(p-1) / 4}$ for $\nu<\nu_{\mathrm{c}}$ and $F \propto t^{-(3 p-2) / 4}$ for $\nu_{\mathrm{c}}<\nu$, respectively (Sari, Piran \& Narayan 1998). By assuming $\alpha_{1}=0.88$, the electron spectral index is estimated as $p=2.17$. Note that, this electron spectral index agrees well with those of electrons accelerated in relativistic shock waves (e.g., Dado, Dar \& Rujula 2003). Furthermore, we expect that power-law slope of the light curve would change from 0.88 to 1.13 for $\nu_{\mathrm{c}}<\nu$. Again, this is approximately consistent with the observed spectral index after $t_{\mathrm{b} 1}$, where $\alpha_{2}=1.18 \pm 0.01$. Therefore, we conclude that the first break in the optical afterglow light curve at $t_{\mathrm{b} 1}$ is the most probably cooling-break where the cooling frequency crosses down the observed optical frequency.

Under this assumption, we can determine important physical parameters for the GRB emission. For example, we can estimate $\epsilon_{B}$ and $\epsilon_{e}$, where $\epsilon_{B}$ and $\epsilon_{e}$ are the fractions of the shock energy given to magnetic field and electrons at the shock (Sari, Piran \& Narayan 1998). In case of slow cooling, $t_{m}<t_{R}<t_{c}$ would be expected. Since we started our observation 0.047 days after the burst, we can limit the range of $t_{m}$ as $t_{m}<t_{o b s}=0.047$ days. For $t_{c}=t_{\mathrm{b} 1}=0.26$ days, $t_{m}<0.047$ days, $E=10^{52} \mathrm{erg}, n=1 \mathrm{~cm}^{-3}, \nu=0.5 \times 10^{15}$ $\mathrm{Hz}$, we obtain

$$
\begin{aligned}
\epsilon_{B} & \sim 0.05\left(\frac{t_{c}}{0.26}\right)^{-\frac{1}{3}}\left(\frac{E_{52}}{1.0}\right)^{-\frac{1}{3}}\left(\frac{n_{1}}{1.0}\right)^{-\frac{2}{3}}\left(\frac{\nu_{15}}{0.5}\right)^{-\frac{2}{3}} \\
\epsilon_{e} & <0.20\left(\frac{\epsilon_{B}}{0.05}\right)^{-\frac{1}{4}}\left(\frac{t_{m}}{0.047}\right)^{\frac{3}{4}}\left(\frac{E_{52}}{1.0}\right)^{-\frac{1}{4}}\left(\frac{\nu_{15}}{0.5}\right)^{\frac{1}{2}}
\end{aligned}
$$

We can also constrain the peak time of reverse shock $t_{A}$ days after the burst (Sari \& Piran 1999).

$$
t_{A} \sim 0.03\left(\frac{\epsilon_{B}}{0.05} \times \frac{1}{0.1}\right)^{-3}\left(\frac{E_{52}}{1.0}\right)^{-1}\left(\frac{n_{1}}{1.0} \times \frac{\nu_{c, 15}}{0.5}\right)^{-2}
$$

The Lorentz factor depends on time, $\gamma(t) \sim\left(3 E / 256 \pi n m_{p} c^{5} t^{3}\right)^{1 / 8}$ (Piran 1999). And the magnetic field is calculated using $\epsilon_{B}$ and the Lorentz factor by $B=\gamma c \sqrt{32 \pi \epsilon_{B} n m_{p}}$. Assuming $E=10^{52} \mathrm{erg}$, the Lorentz factor and the magnetic field strength at two characteristic 
breaks time are $\gamma=9.7, B=0.86$ gauss at $t_{\mathrm{b} 1}=0.26$ days and $\gamma=7.4, B=0.64$ gauss at $t_{\mathrm{b} 2}=0.54$ days, respectively.

The values of $\epsilon_{B}$ and $\epsilon_{e}$ estimated in the preceding chapter are in good agreement with the averages of these parameters for GRBs calculated by Panaitescu \& Kumar (2001), which are $\log \epsilon_{B}=-2.4 \pm 1.2$ and $\epsilon_{e}=0.062 \pm 0.045$.

\subsection{Bump at $t \sim 0.08-0.09$ days}

Finally, we comment on a small "bump" of the light curves at $t \sim 0.08-0.09$ days ( $\left.t_{\text {bump }}\right)$ with an amplitude of $\sim 0.1$ mag. Uemura et al. (2003) reported a change of slopes from 0.74 to 0.95 days at $t=0.085$ days. However our earliest data at $t<0.08$ days has a slope steeper than 0.74 . The light curve at $t>0.09$ days lies on the extrapolation of this earliest segment. We consider this feature as a bump rather than a break.

Short time variabilities, i.e., "bumps and wiggles", may be associated with the forward/reverse shock structures along the afterglow emitting regions (Kobayashi \& Zhang 2003), repeated energy injection from the central engine, or fluctuation in the density of the interstellar medium (Nakar, Piran \& Granot 2003).

First, we can rule out a case with the forward/reverse shock structure, since it predicts the light curve should not have the same power-law index before and after the bump. A case with repeated energy injection is also ruled out since after the injection the light curve after the bump should have the same power-law decay slope, but with a larger normalization. Therefore, we conclude that the bumps in the light curve is likely due to the fluctuation in the external density of the interstellar medium (Nakar, Piran \& Granot 2003).

We can estimate the distance from the central engine $R(t)$ (Piran 1999) and the density variation (Nakar, Piran \& Granot 2003) at $t_{\text {bump }}$.

$$
\begin{gathered}
R(t) \sim 2.2 \times 10^{17}\left[3\left(\frac{E_{52}}{1.0}\right)\left(\frac{t(\mathrm{sec})}{7300}\right) / \pi m_{p} c\left(\frac{n_{1}}{1.0}\right)\right]^{\frac{1}{4}} \mathrm{~cm} \\
\left(n / n_{0}\right) \sim 1.1 \times\left[\left(F_{\nu} / F_{0}\right) / 1.1\right]^{4 /(1+p)}
\end{gathered}
$$

We find that density is enhanced about $10 \%$ at the distance of $2.2 \times 10^{17} \mathrm{~cm}$. 


\section{Summary}

We observed extremely bright optical afterglow of GRB 030329 from 67 minutes after the burst. Our observational results show that the shocked electrons are in the slow cooling regime with an electron index of 2.17 in this burst, and that the burst occurred in a uniform ISM, that is, GRB 030329 can be understood very well in the predicted "standard" model. We conclude the first break changes the power law index by $\sim 0.3$, consistent with the cooling-break in the frame work of the standard external shock model.

We are grateful to the other members of the HETE-2 Ops Team for providing the location of GRB 030329. We are also grateful to R. Burenin and his collaborators for kindly

providing us the numerical values of the filtered data and to K. Torii for giving useful advises on photometry. This work is supported by the Grants-in-aid for Scientific Research Program from the Ministry of Education, Science and Culture of Japan to NK.

\section{REFERENCES}

Akerlof, C. W. \& McKay, T. A. 1999, GRB Circ. 205

Beuermann, K. et al. 1999, A\&A, 352, L26

Burenin, R. A. et al. 2003, Astronomy Letters, 29, 573

Cantiello, M. et al. 2003, GRB Circ. 2074

Chevalier, R. A. \& Li, Z. 1999, ApJ, 520, L29

Dado, S., Dar, A., \& De Rújula, A. 2003, ApJ, 594, L89

Fitzgerald, J. B. \& Orosz, J. A. 2003, GRB Circ. 2056 \& 2070

Fox, D. W. 2002, GRB Circ. 1564

Fox, D. W. \& Price, P. A. 2002, GRB Circ. 1731

Galama, T. J. et al. 1998, Nature, 395, 670

Granot, J., Nakar, E., \& Piran, T., 2003, astro-ph/0304563

Greiner, J. et al. 2003, GRB Circ. 2020

Henden, A. 2003, GRB Circ. 2023 
Hjorth, J. et al. 2003, Nature, 423, 847

Ibrahimov, M. A. et al. 2003, GRB Circ. 2077

Iwamoto, K. et al. 1998, Nature, 395, 672

Kawabata, K. S. et al. 2003, ApJ, 593, L19

Klose, S., Hoegner, C., \& Greiner, J. 2003, GRB Circ. 2029

Kobayashi, S. \& Zhang, B. 2003, ApJ, 582, L75

Li, W., Chornock, R., Jha, S., \& Filippenko, A. V. 2003, GRB Circ. 2064

Lipkin, Y., Ofek, E. O., \& Gal-Yam, A. 2003, GRB Circ. 2034

Meegan, C. A. et al. 1992, Nature, 355, 143

Nakar, E., Piran, T., \& Granot, J. 2003, New Astronomy, 8, 495

Panaitescu, A., Meszaros, P., \& Rees, M. J. 1998, ApJ, 503, 314

Panaitescu, A. \& Kumar, P. 2001, ApJ, 560, L49

Pavlenko, E. et al. 2003, GRB Circ. 2050 \& 2067

Peterson, B. A. \& Price, P. A. 2003, GRB Circ. 1985

Piran, T. 1999, Phys. Rep., 314, 575

Price, A. 2003, GRB Circ. 2058

Price, P. A. et al. 2003, Nature, 423, 844

Price, A. \& Mattei, J. 2003, GRB Circ. 2071

Rhoads, J. E. 1999, ApJ, 525, 737

Ricker, G. R., Vanderspek, R. K., \& HETE Science Team, 2002, American Astronomical Society Meeting, 201,

Rumyantsev, V. et al. 2003, GRB Circ. 2028

Sari, R., Piran, T., \& Narayan, R. 1998, ApJ, 497, L17

Sari, R., Piran, T., \& Halpern, J. P. 1999, ApJ, 519, L17 
Sari, R. \& Piran, T. 1999, ApJ, 520, 641

Stanek, K. Z., Martini, P., \& Garnavich, P. 2003, GRB Circ. 2041

Stanek, K. Z. et al. 2003, ApJ, 591, L17

Tiengo, A. et al. 2003, A\&A, 409, 983

Torii, K. 2003, GRB Circ. 1986

Uemura, M. et al. 2003, Nature, 423, 843

Vanderspek, R. et al. 2003, GRB Circ. 1997 


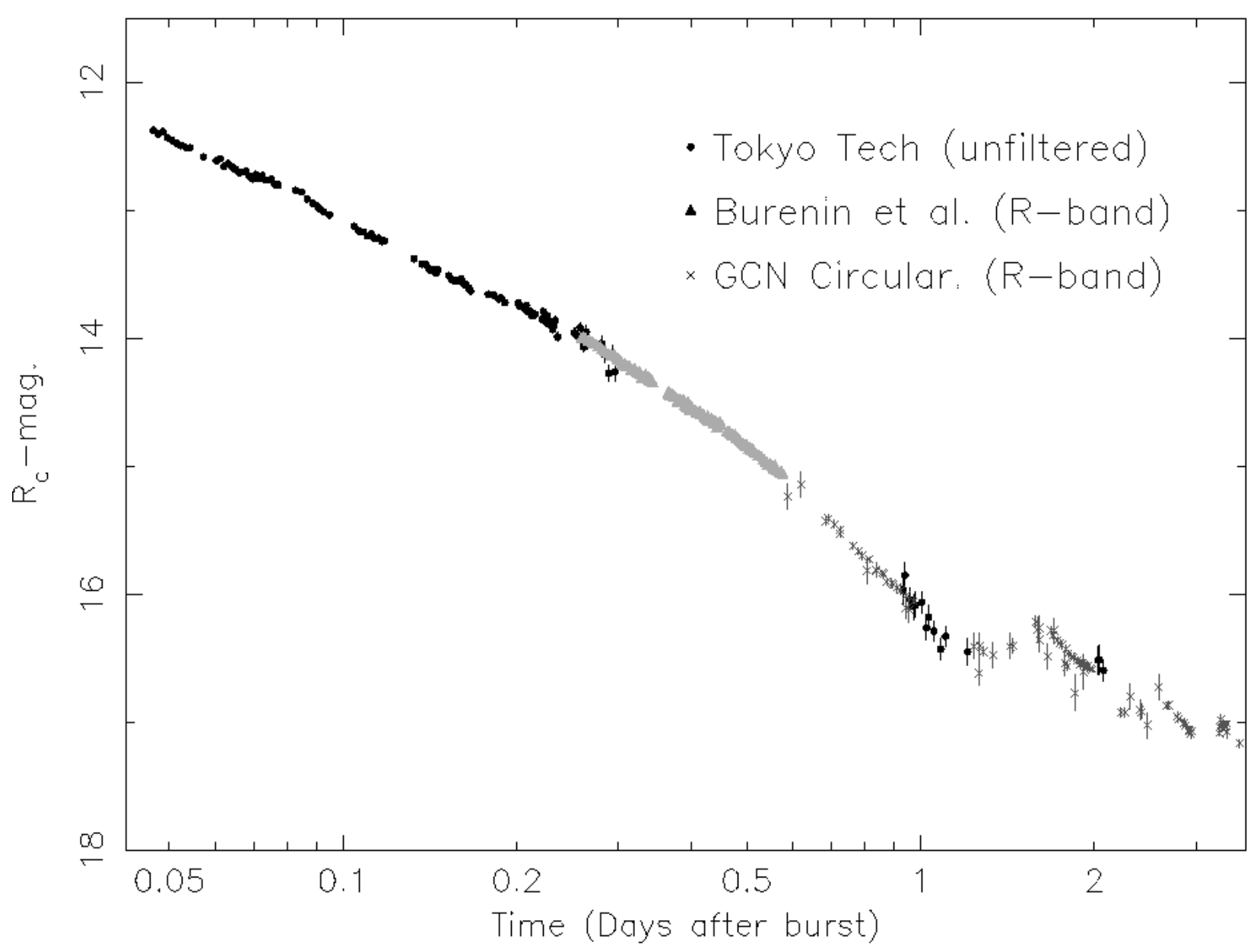

Fig. 1. - Light curve of the optical afterglow of GRB 030329. The filled circles are our observations, the filled triangles come from Burenin et al. (2003), and the rest comes from GCN 2028, 2029, 2034, 2041, 2050, 2056, 2058, 2064, 2067, 2070, 2071, 2074, 2077, and KAIT. These magnitudes were translated using the standard sequence by Henden (2003). 


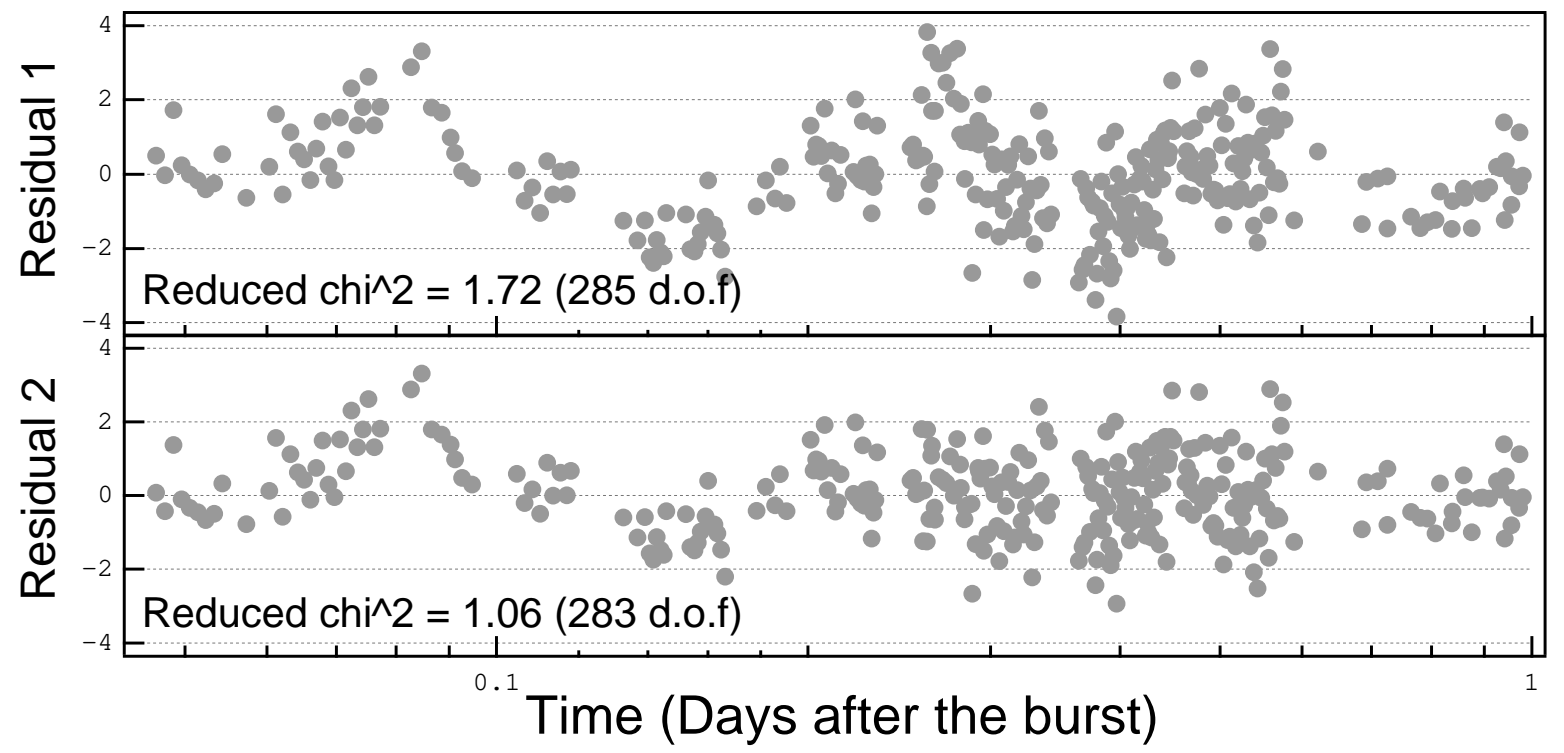

Fig. 2.- Residuals of the light curve of the optical afterglow for the two models. top; the residual from a broken power-law which is containing a single, broad break. bottom; the residual from a double broken power-law (see text). 
Table 1. Predicted decay slopes for various theoretical models

\begin{tabular}{clcc}
\hline \hline Model & Environment & $\alpha$ & Comment \\
\hline$(1) \nu_{\mathrm{m}}<\nu_{\mathrm{c}}<\nu_{\mathrm{R}}$ & ISM & 0.49 & $\alpha$ and $\beta$ are inconsistent \\
& Wind & 0.49 & $\alpha$ and $\beta$ are inconsistent \\
$(2) \nu_{\mathrm{m}}<\nu_{\mathrm{R}}<\nu_{\mathrm{c}}$ & ISM & 0.99 & O.K. \\
& Wind & 1.49 & $\alpha$ does not fit data \\
$(3) \nu_{\mathrm{R}}<\nu_{\mathrm{m}}<\nu_{\mathrm{c}}$ & ISM & - & $\alpha<0$ \\
& Wind & - & $\alpha<0$ \\
$(4) \nu_{\mathrm{c}}<\nu_{\mathrm{m}}<\nu_{\mathrm{R}}$ & ISM & 0.49 & $\alpha$ and $\beta$ are inconsistent \\
& Wind & 0.49 & $\alpha$ and $\beta$ are inconsistent \\
$(5) \nu_{\mathrm{c}}<\nu_{\mathrm{R}}<\nu_{\mathrm{m}}$ & ISM & 0.25 & $\alpha$ does not fit data \\
& Wind & 0.25 & $\alpha$ does not fit data \\
$(6) \nu_{\mathrm{R}}<\nu_{\mathrm{c}}<\nu_{\mathrm{m}}$ & ISM & - & $\alpha<0$ \\
& Wind & - & $\alpha<0$ \\
\hline
\end{tabular}

